\title{
Rapidly enlarging low-grade fibromyxoid sarcoma with intracranial extension in a 5-year-old girl: case report
}

\author{
Ian K. White, MD, ${ }^{1}$ Andrea G. Scherer, MD, ${ }^{1}$ Maraya M. Baumanis, BS, ${ }^{1}$ Marwah Abdulkader, MD, ${ }^{2}$ \\ and Daniel H. Fulkerson, MD³

\begin{abstract}
Departments of ${ }^{1}$ Neurological Surgery and ${ }^{2}$ Pathology and Laboratory Medicine, Indiana University School of Medicine; and ${ }^{3}$ Department of Neurological Surgery, Division of Pediatric Neurosurgery, Goodman Campbell Brain and Spine, Indiana
\end{abstract} \\ University School of Medicine, Indianapolis, Indiana
}

\begin{abstract}
Low-grade fibromyxoid sarcoma (LGFMS) is a rare mesenchymal tumor that is characterized by a benign histology but potentially aggressive clinical behavior, with a high rate of recurrence and metastasis. It primarily occurs in young adults in the extremities, inguinal area, neck, or chest wall. There are rare reports of intracranial LGFMS in adults. In this report, the authors present the case of a 5-year-old girl who presented with a rapidly enlarging frontal scalp mass. Pathological examination of the resected mass demonstrated LGFMS. To the authors' knowledge, this is the only reported case of intracranial LGFMS in a child.
\end{abstract}

http://thejns.org/doi/abs/10.3171/2015.3.PEDS14564

KEY WORDS fibromyxoid sarcoma; brain tumor; pediatric; mesenchymal; oncology

$\mathrm{L}$ OW-GRADE fibromyxoid sarcoma (LGFMS) is a mesenchymal, soft tissue-tumor that is characterized by a benign histological appearance but aggressive clinical behavior. ${ }^{3,4}$ This tumor occurs primarily in young to middle-aged adults and is only rarely seen in children. The most common anatomical locations are the lower extremities, thorax, inguinal area, and upper limbs..$^{15}$ There are 2 prior cases in the English literature describing intracranial involvement in adults..$^{10,13}$

LGFMS presents as a painless enlarging mass. Histologically, this neoplasm consists of contrasting fibrous and myxoid areas with a swirling, whorled growth pattern, and bland, benign-appearing fibroblastic spindle cells. Initially a controversial diagnosis, LGFMS is now more clearly defined by immunohistochemistry and molecular analyses.

To our knowledge, intracranial involvement of LGFMS in a child has not been previously described. We report our experience and follow-up data in a case involving a 5 -year-old child with a rapidly expanding mass with both intra- and extracranial components.

\section{Case Report}

History and Examination

An otherwise healthy 5-year-old girl presented to Riley Hospital for Children (Indianapolis, Indiana) with a nontender, enlarging scalp mass. The mass had developed over a period of 2 months and seemed to be growing rapidly. The remainder of her physical examination and review of systems findings were normal.

\section{Imaging}

An ultrasound examination of the patient's head was performed at the referring hospital. This showed that the mass extended into the intracranial space. CT scan demonstrated a large, extraaxial mass with both an extracranial and an intracranial component (Fig. 1 left). There was destruction of the skull at the level of the coronal suture (Fig. 1 right).

The mass measured $6.8 \times 6.1 \times 6.4 \mathrm{~cm}$ on MRI. It was hyperintense on T2-weighted images (Fig. 2A), showed

ABBREVIATIONS LGFMS = low-grade fibromyxoid sarcoma; PDGF = platelet-derived growth factor .

SUBMITTED October 17, 2014. ACCEPTED March 23, 2015.

INCLUDE WHEN CITING Published online July 3, 2015; DOI: 10.3171/2015.3.PEDS14564.

DISCLOSURE The authors report no conflict of interest concerning the materials or methods used in this study or the findings specified in this paper. 

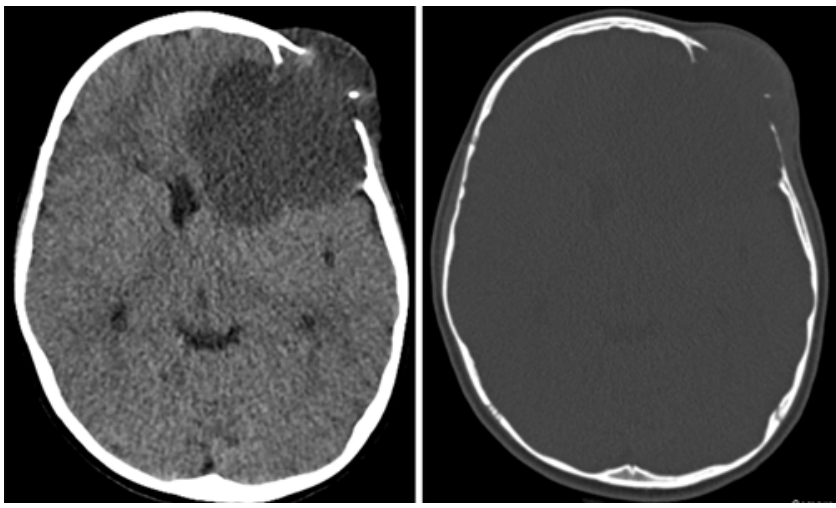

FIG. 1. Left: Noncontrast head CT scan showing a large tumor with intra- and extracranial components. Right: Bone window CT showing local bone destruction.

speckled enhancement on T1-weighted images obtained after administration of contrast medium (Fig. 2B), and increased diffusion on diffusion weighted imaging (Fig. 2C). Spinal MRI did not show any evidence of metastasis. The initial differential diagnosis based on imaging was a primary bone tumor, such as chondroid tumor or atypical osteosarcoma. The imaging findings were not consistent with dermoid, epidermoid, lymphoma, Ewing's sarcoma, or hemangioma.

\section{Operation}

The patient was positioned supine with 3-point head fixation. A large, bicoronal scalp flap was created, allowing access to the lesion and to the contralateral skull for planned autograft cranioplasty (Fig. 3 left). Two bur holes were placed, and a rim of bone was drilled around the mass (Fig. 3 right). The mass had a firm, tan capsule. We were able to develop a plane between the tumor and the surrounding peripheral dura mater. At the depths, the tumor had eroded through the dura and required dissection from the brain parenchyma. We repaired the dura with a patch graft. A right-sided craniotomy was then performed to harvest autograft for a split-thickness bone graft.

\section{Pathological Examination}

The tumor was largely composed of areas of slender, spindle-shaped cells without significant atypia against an alternating background of a myxoid and collagenous matrix (Fig. 4A). The collagenous matrix was characterized by spindle-shaped cells in a fascicular or whorling pattern with little to no mitotic figures in 10 high-power fields (Fig. 4B). The myxoid component was characterized by cells with myxoid/foamy cytoplasm with satellite spindleshaped cells once again without atypia (Fig. 4C). The differential diagnosis included a number of low-grade myxoid tumors, including myxoid chondrosarcoma, myxoid meningioma, myxoid liposarcoma, myxofibrosarcoma, fibromy xoid sarcoma, and myxoma.

Immunohistochemistry studies should lightly positive staining for beta-catenin and more strongly positive staining for vimentin. Studies for progesterone receptors, EMA, S-100 protein, smooth muscle actin, and desmin were all negative. The Ki-67 index was 2\%-3\%. Cytogenetic analyses and karyotyping displayed "ring chromosome" abnormalities resulting from $\mathrm{t}(7,16)(\mathrm{q} 32-34 ; \mathrm{p} 11)$ and the fusion gene FUS-CREB3L2. Analysis of the surrounding tissues showed extension into fibromuscular tissue as well as entrapping the trabeculae of adjacent bone. Due to the negative markers, it was believed that meningioma was less plausible and fibromatosis was unlikely, although the beta-catenin was slightly positive. The invasive nature of the neoplasm pointed more toward an invasive sarcoma rather than a myxoma. The benign appearance of the cells led to the diagnosis of LGFMS.

\section{Clinical Follow-Up}

The patient recovered well from surgery without deficit. Immediate postoperative MRI showed a gross-total resection. The child did not undergo any adjuvant therapy. An imaging study performed 18 months after surgery showed no evidence of tumor recurrence or regrowth (Fig. 5).

\section{Discussion}

LGFMS was first recognized as a unique entity by Evans in 1987. ${ }^{4}$ It was originally described as a slow-growing, asymptomatic soft-tissue tumor with a deceptively benign histology, but with high rate of local recurrence and late metastasis. Subsequent papers by the author described local recurrence in 21 of 33 cases, with a median time to recurrence of 3.5 years. ${ }^{3}$ Metastasis occurred in 15
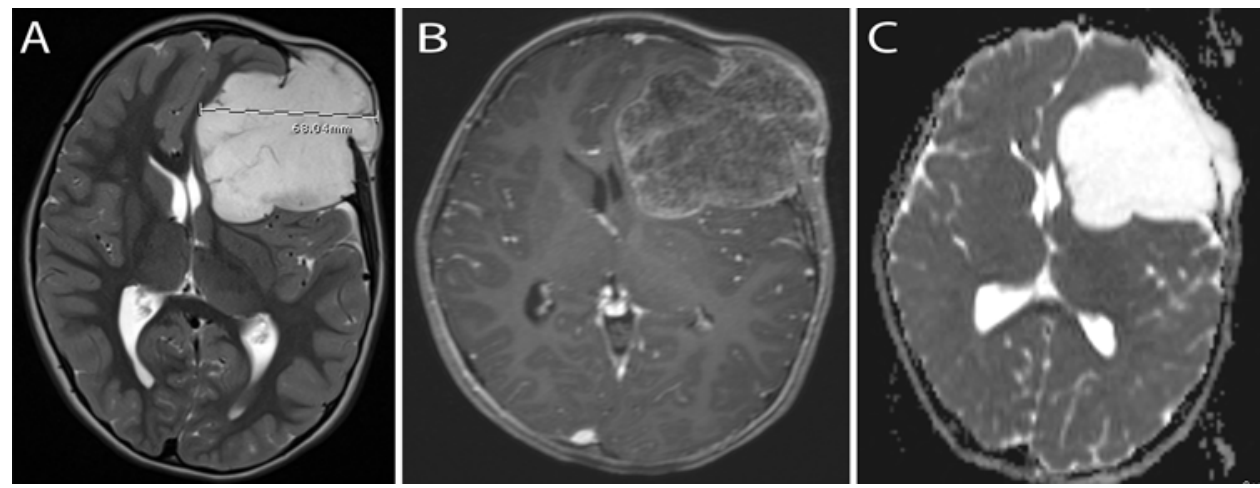

FIG. 2. MR images demonstrating a $6.8 \times 6.1 \times 6.4-\mathrm{cm}$ tumor. The tumor was hyperdense on T2-weighted images $(\mathbf{A})$ with mass effect and midline shift of $5 \mathrm{~mm}$. It showed speckled enhancement on T1-weighted images obtained after administration of a contrast agent (B). There was increased signal intensity on diffusion weighted images (C). 


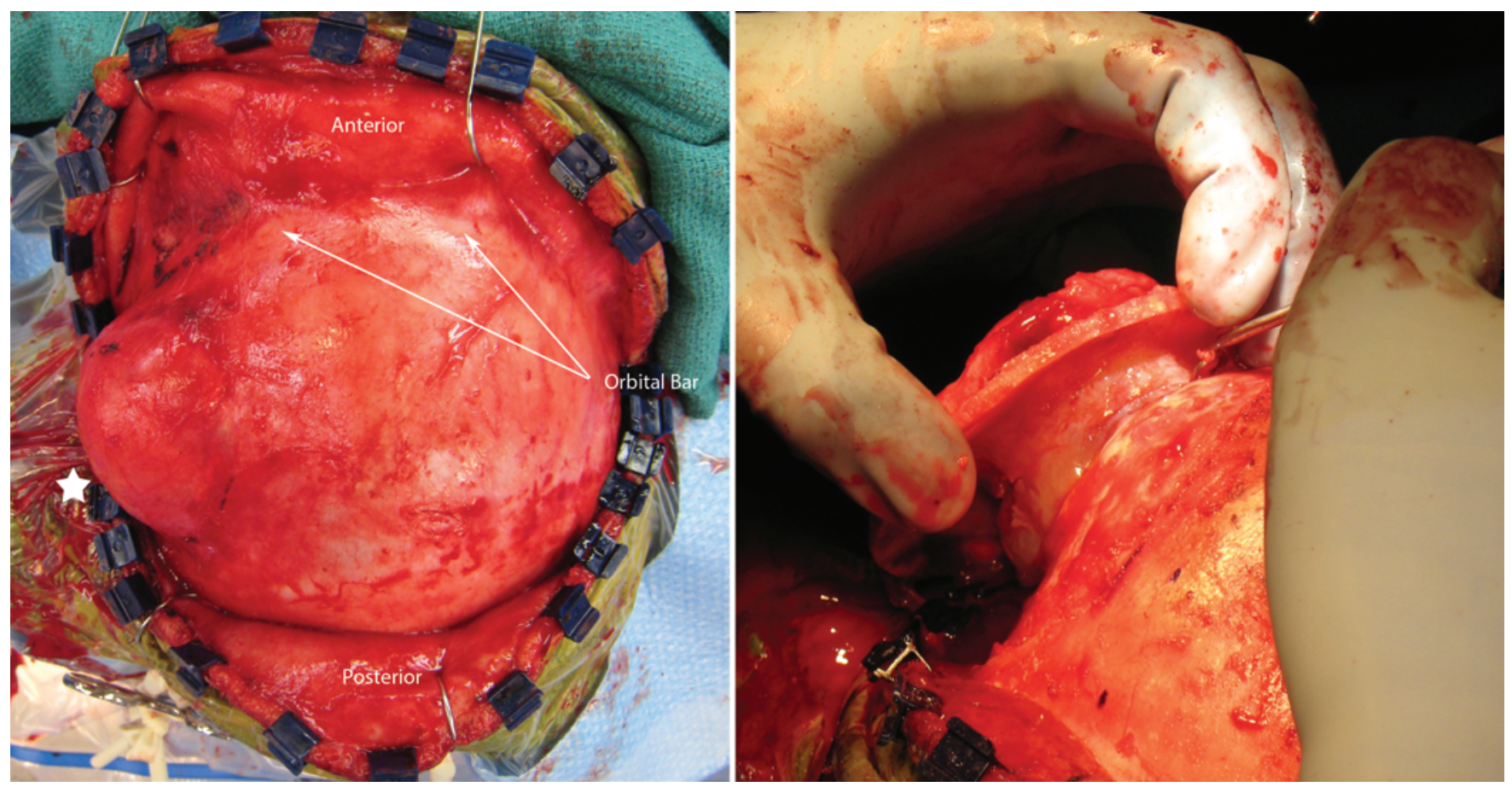

FIG. 3. Intraoperative photographs obtained during tumor resection. A large, bicoronal scalp flap was developed (left) allowing access to the lesion (white star adjacent to lesion) and to the contralateral bone for cranioplasty. A rim of bone around the lesion was excised (right), allowing mobilization of an encapsulated, firm mass.

cases, with a range of $0-45$ years (median 5 years) from time of discovery. Patients had prolonged survival after metastasis discovery (median 15 years). Two patients had de-differentiation of the tumor and died within 1 year.

Other large series show a more benign course. Folpe et al. published a series of 73 cases. In this series, only 5 patients showed recurrence, 3 patients had metastasis, and there was 1 death. ${ }^{5}$ Guillou et al. reported a $10 \%$ recurrence rate and a $5 \%$ metastasis rate in 63 patients with at least 11 years' follow-up. ${ }^{6}$

Previously published cases show that the tumor occurs in young to middle-aged adults, with the primary ana- tomical sites being the deep tissues of the lower extremities, followed by the upper extremities, chest wall, and abdominal cavity. To our knowledge, only 2 cases showing intracranial involvement have been previously published in the English-language literature, and both of these cases involved adults. ${ }^{10,13}$

\section{Pathology}

The pathological diagnosis of LGFMS is challenging, and there are many entities with similar histological appearances. Myxomatous meningioma and chordoid menin-
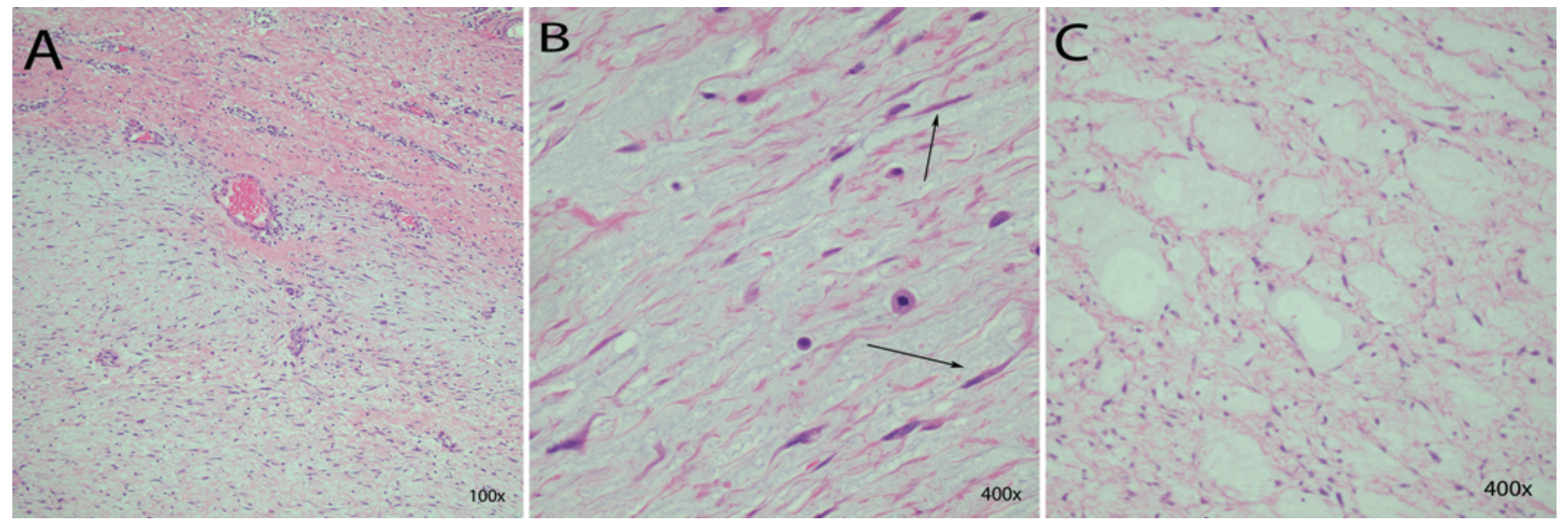

FIG. 4. A: A section of the tumor viewed at low magnification showing a cytoarchitectural matrix of spindle-shaped cells against a myxoid matrix alternating with whirling cells within a collagenous matrix. B: A section viewed at higher magnification showing the collagenous matrix with slender spindle-shaped cells (arrows) without significant atypia. C: A section from a different area of the tumor showing a myxoid/mucoid matrix surrounded by satellite cells without atypia. H\&E, original magnification $\times 100(A)$ and $\times 400$ (B and $\mathrm{C})$. 


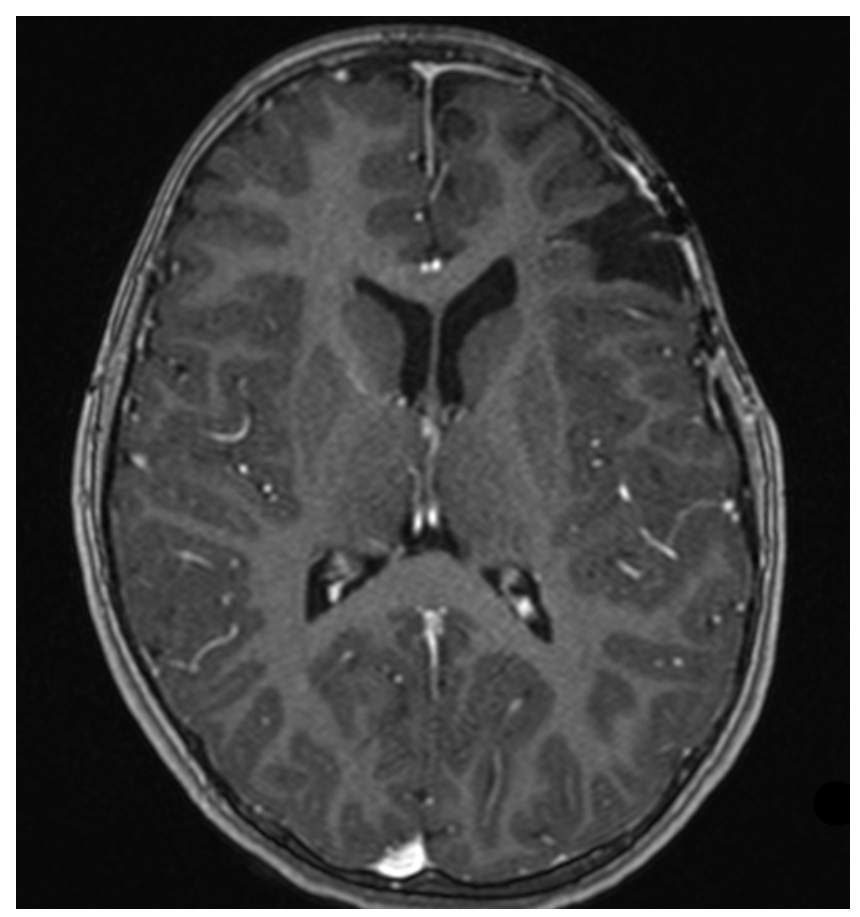

FIG. 5. Axial contrast-enhanced T1-weighted MR images obtained 18 months after surgery showing gross-total resection without evidence of recurrence.

gioma were considered in our patient due to the anatomical location, abundant mucinous cytoplasm, and matrix of the tumor. However, the negative cytokeratin and EMA staining led us away from these diagnoses. ${ }^{13}$ The invasive growth pattern and destruction of bone with entrapment trabeculae is not commonly characteristic of meningioma.

Myxoid chondrosarcoma, liposarcoma, and myxofibrosarcoma were also considered. Chondrosarcoma tends to be positive for vimentin and S-100; this tumor was negative. ${ }^{11-13,16-18}$ The tumor was negative for cytokeratin and smooth muscle actin which, along with vimentin and S-100, are suggestive of liposarcoma. Myxofibrosarcoma show much more cellular atypia than those in this tumor. ${ }^{11-13,16-18}$ Myxoma was also considered. Our patient's tumor had much less collagen and is less cellular than would be expected in a myxoma. Myxoma tends not to be invasive in the surrounding soft tissues.

Molecular analysis may clarify the pathology. LGFMSs bear the translocation $\mathrm{t}(7,16)(\mathrm{q} 32-34 ; \mathrm{p} 11)$ or $\mathrm{t}(11,16)$ (p11;p11) translocations, resulting in FUS-CREB3L2 or FUS-CREB3L1 fusion genes. "Ring chromosomes" are specific to soft-tissue sarcomas and have been described in LGFMS. ${ }^{1,8,9}$ These ring chromosomes are typically composed of translocations from chromosomes 17 and 22 and fuse the platelet-derived growth factor $(P D G F)$ gene to a collagen A1 gene. This creates a fusion gene which, in turn, creates a constitutive activation of the PDGF gene product, which may be involved in the pathogenesis of the tumor. A second abnormality that may lead to ring chromosomes is the fusion gene of FUS-CREB3L2 or FUSCREB3L1, resulting from $\mathrm{t}(7,16)(\mathrm{q} 32-34 ; \mathrm{p} 11)$ or $\mathrm{t}(11,16)$ (p11;p11) translocations. These 2 translocations are highly sensitive and specific for LGFMS. In this case, karyotype and cytogenetic analysis showed both ring chromosome abnormalities resulting from $\mathrm{t}(7,16)(\mathrm{q} 32-34 ; \mathrm{p} 11)$ and the fusion gene FUS-CREB3L2, confirming the suspicion of LGFMS. These genetic studies have been shown to be very specific for diagnosing LGFMS and can be performed on previously treated tissue. This serves as a reasonable option if the diagnosis remains in doubt. .,6,7,16 $^{-}$

\section{Treatment and Follow-Up}

Treatment of LGFMS is surgical, and the extent of resection greatly affects the prognosis. There is some suggestion that the published series with lower recurrence rates had higher rates of initial gross-total resection. . $^{2,3,5,6,18}$ Surgery is the treatment of choice for local recurrence and for metastatic lesions. Other authors have reported treatment with chemotherapy or radiation therapy, but there is no convincing evidence that this alters the outcomes. ${ }^{11-14,16}$ Patients with this diagnosis should be counseled that this tumor could recur throughout their lifetime. Therefore, long-term follow-up is necessary.

\section{Conclusions}

LGFMS is a mesenchymal tumor with a seemingly benign histology but an aggressive clinical course. Resection is the treatment of choice. The authors report a case of a 5-year-old child with a large LGFMS with extra- and intracranial components. To date, only 2 prior cases (both involving adults) have been published showing intracranial LGFMS. The child has done well after resection, with no signs of recurrence in 18 months of follow-up.

\section{References}

1. Bartuma H, Möller E, Collin A, Domanski HA, Von Steyern FV, Mandahl N, et al: Fusion of the FUS and CREB3L2 genes in a supernumerary ring chromosome in low-grade fibromyxoid sarcoma. Cancer Genet Cytogenet 199:143-146, 2010

2. Billings SD, Giblen G, Fanburg-Smith JC: Superficial lowgrade fibromyxoid sarcoma (Evans tumor): a clinicopathologic analysis of 19 cases with a unique observation in the pediatric population. Am J Surg Pathol 29:204-210, 2005

3. Evans HL: Low-grade fibromyxoid sarcoma: a clinicopathologic study of 33 cases with long-term follow-up. Am J Surg Pathol 35:1450-1462, 2011

4. Evans HL: Low-grade fibromyxoid sarcoma. A report of two metastasizing neoplasms having a deceptively benign appearance. Am J Clin Pathol 88:615-619, 1987

5. Folpe AL, Lane KL, Paull G, Weiss SW: Low-grade fibromyxoid sarcoma and hyalinizing spindle cell tumor with giant rosettes: a clinicopathologic study of 73 cases supporting their identity and assessing the impact of high-grade areas. Am J Surg Pathol 24:1353-1360, 2000

6. Guillou L, Benhattar J, Gengler C, Gallagher G, RanchèreVince D, Collin F, et al: Translocation-positive low-grade fibromyxoid sarcoma: clinicopathologic and molecular analysis of a series expanding the morphologic spectrum and suggesting potential relationship to sclerosing epithelioid fibrosarcoma: a study from the French Sarcoma Group. Am J Surg Pathol 31:1387-1402, 2007

7. Maretty-Nielsen K, Baerentzen S, Keller J, Dyrop HB, Safwat A: Low-grade fibromyxoid sarcoma: incidence, treatment strategy of metastases, and clinical significance of the FUS gene. Sarcoma 2013:256280, 2013 
8. Mezzelani A, Sozzi G, Nessling M, Riva C, Della Torre G, Testi MA, et al: Low grade fibromyxoid sarcoma: a further low-grade soft tissue malignancy characterized by a ring chromosome. Cancer Genet Cytogenet 122:144-148, 2000

9. Möller E, Hornick JL, Magnusson L, Veerla S, Domanski HA, Mertens F: FUS-CREB3L2/L1-positive sarcomas show a specific gene expression profile with upregulation of CD24 and FOXL1. Clin Cancer Res 17:2646-2656, 2011

10. Paulus W, Slowik F, Jellinger K: Primary intracranial sarcomas: histopathological features of 19 cases. Histopathology 18:395-402, 1991

11. Rando G, Buonuomo V, D’Urzo C, Vecchio F, Caldarelli M, Pintus C: Fibromyxoid sarcoma in a 4-year-old boy: case report and review of the literature. Pediatr Surg Int 21:311312,2005

12. Rekhi B, Deshmukh M, Jambhekar NA: Low-grade fibromyxoid sarcoma: a clinicopathologic study of 18 cases, including histopathologic relationship with sclerosing epithelioid fibrosarcoma in a subset of cases. Ann Diagn Pathol 15:303-311, 2011

13. Saito R, Kumabe T, Watanabe M, Jokura H, Shibuya M, Nakazato Y, et al: Low-grade fibromyxoid sarcoma of intracranial origin. J Neurosurg 108:798-802, 2008

14. Shidham VB, Ayala GE, Lahaniatis JE, Garcia FU: Lowgrade fibromyxoid sarcoma: clinicopathologic case report with review of the literature. Am J Clin Oncol 22:150-155, 1999
15. Tang Z, Zhou ZH, Lv CT, Qin LY, Wang Y, Tian G, et al: Low-grade fibromyxoid sarcoma: clinical study and case report. J Oral Maxillofac Surg 68:873-884, 2010

16. Vernon SE, Bejarano PA: Low-grade fibromyxoid sarcoma: a brief review. Arch Pathol Lab Med 130:1358-1360, 2006

17. Vlacha V, Repanti M: Low grade fibromyxoid sarcoma and neurofibromatosis type I. Pediatr Blood Cancer 50:938, 2008 (Letter)

18. Winfield HL, De Las Casas LE, Greenfield WW, Santin AD, McKenney JK: Low-grade fibromyxoid sarcoma presenting clinically as a primary ovarian neoplasm: a case report. Int J Gynecol Pathol 26:173-176, 2007

\section{Author Contributions}

Conception and design: Fulkerson, White. Acquisition of data: Fulkerson, Scherer, Abdulkader. Analysis and interpretation of data: Scherer, Baumanis. Drafting the article: all authors. Critically revising the article: Fulkerson, White. Reviewed submitted version of manuscript: Fulkerson. Study supervision: Fulkerson.

\section{Correspondence}

Daniel H. Fulkerson, Indiana University School of Medicine/ Goodman Campbell Brain and Spine, Riley Hospital for Children, 702 Barnhill Dr. \#1134, Indianapolis, IN 46202-5200. email: dfulkers@iupui.edu. 